\title{
Lesões por esforços repetitivos/distúrbios osteomusculares relacionados ao trabalho de cirurgiões-dentistas: aspectos biomecânicos
}

\author{
Gilsée Ivan Regis Filho UFSC \\ Glaycon Michels UFSC \\ Ingeborg Sell UFSC
}

\section{RESUMO}

As patologias denominadas genericamente Lesões por Esforços Repetitivos/Distúrbios Osteomusculares Relacionados ao Trabalho (LERs/DORTs) e que apresentam sinais e sintomas de inflamações dos músculos, tendões, fáscias e nervos dos membros superiores, cintura escapular e pescoço, entre outros, têm chamado a atenção não só pelo aumento de sua incidência mas por também existirem evidências de sua associação com o ritmo de trabalho. 0 exercício profissional obriga que cirurgiões-dentistas utilizem na execução de suas tarefas os membros superiores e estruturas adjacentes, frequentemente com repetitividade de um mesmo padrão de movimento, compressão mecânica das estruturas localizadas na região e a adoção de posturas incorretas, utilizando força excessiva e, na maioria dos casos, trabalhando, ainda, sob a pressão do tempo de realização. O presente estudo utilizou a análise biomecânica, através da cinemetria e da eletromiografia, para buscar evidências da relação entre as tarefas executadas pelo cirurgião-dentista e as LERs/DORTs. A análise dos dados cinemáticos revelou que as principais atividades realizadas pelos cirurgiõesdentistas submetem duas ou mais regiões do corpo do profissional a posturas consideradas de risco médio e alto, sendo que a análise dos sinais eletromiográficos revelou um grande comprometimento dos grupamentos musculares flexores e extensores do carpo e do trapézio. Finalmente, o presente estudo é mais um indicativo de que a atividade desses profissionais os expõe a risco considerável de adquirir LERs/DORTs.

\section{Cumulative trauma disorders/work related musculoskeletal disorder affecting surgeon-dentists: biomechanical aspects}

\begin{abstract}
Pathologies generally named Cumulative Trauma Disorders/Work Related Musculoskeletal Disorders (CTDs/WRMDs), presenting signs and symptoms of inflammation of muscles, tendons, fascia and nerves of the upper members, shoulder girdle and neck, among others, have been observed not only on account of their incidence but also their association with work rhythms. Professional activities oblige dental surgeons to use, during their treatment procedures, the upper body and adjacent structures, where frequently the same pattern of movement is repeated, or mechanical compression of structures in that area occur, due to the adoption of incorrect postures and the use of excessive force, exacerbated in most cases due to time pressures. This study used biomechanical analysis to look for evidence of a relationship between those tasks a dental surgeon performs and the CTDs/WRMDs. The analysis of video data revealed that the main activities dental surgeons perform, force two or more regions of the body to adopt postures considered to be of medium or high risk, and the analysis of electromyographic readings showed that the flexor and extensor muscle groups of the carpi and trapezius are subject to great risk. Finally, the present study is a further indication that the tasks carried out expose the professional to a considerable risk of acquiring CTDS/WRMDs.
\end{abstract}

\section{KEYWORDS}

Repetitive strain injury, cumulative trauma disorders, work related musculoskeletal disorder. 


\section{INTRODUÇÃO}

Com o crescimento significativo da industrialização e da produtividade, aumentaram de maneira bastante evidente os relatos de Lesões por Esforços Repetitivos/Distúrbios Osteomusculares Relacionados ao Trabalho (LERs/DORTs), a ponto de essas patologias serem consideradas uma nova epidemia industrial (FERGUSON, 1987). As patologias denominadas genericamente LERs/DORTs apresentam, entre outros, sinais e sintomas de inflamações dos músculos e/ ou tendões e/ou fáscias e/ou nervos dos membros superiores e/ou cintura escapular e/ou pescoço que têm chamado
Recentemente se observou um aumento da incidência de LERs/DORTs entre intérpretes de linguagem de sinais devido a uma interação entre as exigências da função e o estilo de trabalho, com mais estresse biomecânico sobre as extremidades superiores. Aqueles com sintomatologia dolorosa faziam, geralmente, menos pausas recreativas, apresentavam desvios mão/punho fugindo da posição neutra, moviam mãos/dedos mais rapidamente e possuíam maior tendência para relatar dor (FEUERSTEIN; FITZGERALD, 1992).

No Brasil, a partir de 1985 também houve um aumento significativo dos casos de LERs/DORTs como doença profissional: de $4,69 \%$, naquela data, para $41,77 \%$ em 1988 , sendo que as mulheres são mais facilmente afetadas, talvez devido à acumulação da jornada doméstica, menor número de fibras musculares, menor capacidade de armazenar e converter glicogênio em energia útil e por serem destinadas a elas, na indústria, as atividades repetitivas que exi-

a atenção não só pelo aumento de sua incidência mas por existirem evidências de sua associação com o ritmo de trabalho. Essas patologias em geral não são de tratamento difícil mas apresentam uma evolução ruim, causando dor, perda de força e edemas, sendo responsáveis por uma porcentagem significativa da queda da performance no trabalho.

Normalmente se encontram entre as causas de LERs/ DORTs atividades profissionais que exigem força excessiva com as mãos, posturas inadequadas dos membros superiores, repetitividade de um mesmo padrão de movimento, compressão mecânica das estruturas dos membros superiores e regiões anexas e tempo insuficiente para a realização de determinadas tarefas (REGIS FILHO; LOPES, 1997).

Por outro lado, estão também entre as atividades causadoras de LERs/DORTs tarefas domésticas que exigem muito das mãos como, por exemplo, jardinagem, tricotar, costurar, fazer crochê, ou o uso de instrumentos musicais como piano, assim como práticas esportivas que exigem grande esforço dos membros superiores, como vôlei, tênis e golfe (McKEAG, 1991). Usuários de terminais de vídeo também constituem geralmente um grupo de risco (ROCHA; PAES; SOBANIA, 1986), pois estudos realizados sobre as condições de trabalho de operadores desses terminais demonstraram que a frequência de queixas quanto aos problemas musculares e visuais são menores onde a organização do trabalho permite certa autonomia em relação à execução das tarefas e onde há a observação de pausas intercaladas às tarefas de natureza diferente - fatores físicos e ambientais estariam por trás do aparecimento dos sintomas (SALORD, 1979, citado por MACIEL, 1985). gem maior habilidade. O quadro se agrava, principalmente, durante a gestação e a menopausa (FACCI et al., 1989; OLIVEIRA, 1991; COUTO, 1994).

Para Couto (1994), se algum trabalhador tem alguma doença do trabalho ou ocupacional que o incapacite, há mais de $1 / 3$ de chance dessa doença decorrer de LERs/ DORTs. Para tanto, basta que exista conjugação dos fatores de risco relacionados à função desse trabalhador, como fatores biomecânicos, psicossociais, administrativos e outros que contribuam para a ocorrência dessas patologias. Assim, as LERs/DORTs como doença ocupacional devem ser analisadas dentro do trinômio homem, máquina e ambiente de trabalho.

Fatores econômico-financeiros e de produtividade obrigam os digitadores a um excesso de toques por hora, entre 10 e 15.000, por exemplo, quando os tendões não toleram mais que 2.000, e o aumento da jornada de trabalho, com a prática do duplo emprego, faz com que $20 \%$ dos digitadores venham a desenvolver algum tipo de LER/DORT (FACCI et al., 1989). Pessoas que executam tarefas altamente repetitivas e forçadas têm 29 vezes mais risco de desenvolver essas patologias nos punhos e mãos (OLIVEIRA, 1991; BARREIRA, 1994; ASSUNÇÃO, 1997; CODO; ALMEIDA, 1997; INSS, 1998).

$\mathrm{Na}$ avaliação de alguns estudos (CUNHA et al., 1992; ERDIL; DICKERSON; GLACKIN, 1994) as principais ocupações associadas às LERs/DORTs são: 1) trabalhadores que atuam na montagem de aviões, de carros e na eletrônica; 2) engarrafadores, empacotadores e transportadores; 3 ) trabalhadores que atuam na produção de carvão (coque), 
na construção civil, indústrias de pneus e da borracha; 4) polidores, açougueiros, caixas, motoristas, inspetores, mineiros, músicos, secretárias, datilógrafos e garçonetes; 5) cortadores e costureiras de tecidos, preparadores de alimentos, fabricantes de malas e processadores de carnes e aves; 6) verificadores de gêneros alimentícios e fabricantes de produtos de tecelagem; 7) fabricantes de metais, forradores de poltronas e usuários de ferramentas vibratórias; 8) profissionais que trabalham em jornais e na produção de artigos oftalmológicos; e 9) trabalhadores dos correios, intérpretes de linguagem de sinais e usuários de terminais de vídeo.

Parece claro o caráter diversificado da epidemiologia da doença quanto aos grupos profissionais atingidos, porém os cirurgiões-dentistas praticamente não são citados na literatura científica. Tal fato ocorre em virtude de as pesquisas e os dados epidemiológicos disponíveis associados à patologia entre os profissionais referidos serem escassos (SILVERSTEIN; FINE; ARMSTRONG, 1987). Por outro lado, somente alguns poucos autores nas últimas três décadas têm relacionado os procedimentos odontológicos com o aparecimento de LERs/DORTs em cirurgiões-dentistas e higienistas dentais. Entre esses autores se encontram Eccles e Powell (1967) que estudaram 231 cirurgiões-dentistas no País de Gales e constataram que 5\% deles trabalhavam em pé e que a maioria referia fadiga apreciável. Do mesmo modo, para Eccles e Daves (1971), a maneira de empunhar instrumentos odontológicos tem grande importância ergonômica, já que modos incorretos levam à tensão muscular.

Para Lawrence (1972) é bastante comum entre cirurgiões-dentistas a degeneração dos discos intervertebrais da região cervical. Outras patologias, como a periartrite escápulo-umeral ou bursite, a hipertrofia muscular no membro mais utilizado e a contratura muscular fisiológica são comuns nesta categoria profissional (MEDEIROS, 1979, citado por SAQUY; PÉCORA, 1996). Encontra-se, também, em vários cirurgiões-dentistas, desigualdade na altura do ombro, inflamação das bainhas tendinosas e artrite das mãos (NOGUEIRA, 1983).

Bauer (1985) apresenta um estudo de caso de Síndrome do Túnel do Carpo em um higienista dental, concluindo que está se tornando uma prática um pouco mais comum o reconhecimento pela classe médica do caráter ocupacional dessa patologia, porém para Holtzhausen (1986) são necessárias mais pesquisas antes de se propor medidas preventivas.

Entretanto, para Barnhart et al. (1991), instrumentos vibratórios e movimentos contínuos contribuem para o surgimento da Síndrome do Túnel do Carpo em cirurgiõesdentistas e higienistas dentais, o que está de acordo com o que sustenta Jetzer (1991), para quem o uso desses instrumentos, assim como os utilizados no desgaste de rebarbas de acrílico, no acabamento de próteses totais, desempenharia papel importante no desencadeamento da síndrome.

Schlim (1990), citado por Lopes e Villanacci Neto (1994), também afirma que há uma grande incidência da Síndrome do Túnel do Carpo entre cirurgiões-dentistas, principalmente periodontistas e endodontistas e naqueles que praticam exodontias em grande número, e entre higienistas dentais.

Para um cirurgião-dentista, a repetição de gestos e a manutenção de contrarresistência, bem como a postura inadequada dos membros superiores, em atividades que exigem constante flexão e extensão do punho, e a compressão mecânica das bases das mãos na utilização de instrumentos periodontais e endodônticos curtos e inadequados são causas ocupacionais imediatas da Síndrome do Túnel do Carpo (BAUER, 1985; JETZER, 1991).

Outro fator a ressaltar, segundo Pece (1995), é que são comuns os casos de inadequação operador/equipamento/ instrumento nas áreas biológicas, obrigando ao profissional a assumir posturas incorretas de trabalho e, consequentemente, na execução da tarefa ocorrem microtraumatismos, ou seja, a destruição de um pequeno número de células - a somatória desses eventos pode originar tecnopatias odontológicas, entre elas as LERs/DORTs.

Estudo realizado por Regis Filho e Lopes (1997) entre profissionais cirurgiões-dentistas participantes do II Encontro de Ex-alunos do Curso de Graduação em Odontologia da Universidade Federal de Santa Catarina - 1996 encontrou $25,81 \%$ de homens e 52,94\% de mulheres com sintomatologia de LERs/DORTs, sendo que ombros e punhos, ambos com $44,44 \%$ cada, eram os locais onde os homens relatavam maior sintomatologia dolorosa. De acordo com os autores, $31,57 \%, 36,84 \%$ e $26,32 \%$ das mulheres apresentavam dor no punho, ombro e dedos, respectivamente.

Números semelhantes são encontrados no trabalho de Santos Filho (1998), onde 58\% dos cirurgiões-dentistas do Serviço Público de Saúde de Belo Horizonte (MG) relataram dor global, sendo que $22 \%, 21 \%, 20 \%$ e $17 \%$ apresentavam dor, respectivamente, no membro superior, coluna, pescoço e ombro.

Diversos autores como Cândido, Bittencourt e Regis Filho (2003), Martins, Fadel e Regis Filho (2003), Fadel et al. (2006a) e Fadel et al. (2006b) apresentam relatos de caso de LERs/DORTs, respectivamente, de Cisto Sinovial de Punho, Síndrome do Túnel Carpal, Dedo em Gatilho e Síndrome do Impacto em cirurgiões-dentistas, sendo todos do sexo feminino nos quais a prática odontológica contribuiu, decididamente, para o desencadeamento da LER/ DORT, ocasionando forte limitação funcional nos dois últimos casos 
Estudo realizado por Regis Filho, Michels e Sell (2006), utilizando o método epidemiológico transversal para buscar evidências da relação entre as tarefas executadas pelo cirurgião-dentista e as LERs/DORTs, revelou presença de associação estatisticamente significante entre os dois sexos e as patologias. Para os autores, o sexo feminino apresentou mais lesões que o masculino, sendo que ombro/braço $(39,40 \%)$, punho/mão $(18,30 \%)$ e pescoço $(17,20 \%)$ são as regiões mais afetadas.

Por outro lado, pesquisas recentes associando as LERs/ DORTs com diversas ocupações, como as realizadas por Murofuse e Marziale (2005), Maciel, Fernandes e Medeiros (2006) e Picoloto e Silveira (2008), com trabalhadores de enfermagem, indústria têxtil e indústria metalúrgica, respectivamente, encontraram elevada incidência dessas patologias, bem como a sua associação com diversos fatores relacionados ao trabalho. Entretanto, três pesquisas realizadas com profissionais da odontologia também indicam risco de as atividades dessa profissão levarem ao desenvolvimento de algum tipo de LER/DORT.

A primeira delas, realizada por Michelotto, Silva Junior e Sydney (2005) com endodontista, utilizando Análise Ergonômica do Trabalho e o Método OWAS, encontrou posturas constrangedoras para o organismo durante $\mathrm{o}$ atendimento clínico.

A segunda delas foi feita por Sayegh et al. (2005), com profissionais libaneses, e indicou presença de dor na coluna lombar de $66 \%$ deles, na coluna cervical de $59 \%$ e no ombro e braço de $62 \%$ dos entrevistados.

A terceira foi realizada por Leggat e Smith (2006) e indicou que $89,1 \%$ dos profissionais tinham experimentado algum sintoma de LER/DORT nos últimos 12 meses, sendo que $57,5 \%$ indicaram o pescoço como a região dolorosa, $53,7 \%$, a região baixa do dorso e $53,3 \%$, o ombro.

Pesquisa realizada por Pietrobon e Regis Filho (2006) em que foi utilizado exame para Análise Postural Corporal com o auxílio de simetógrafo acusou associação estatística entre os perfis sociossanitário e epidemiológico e a análise postural, demonstrando existir ligação estatiscamente significante entre a profissão de cirurgião-dentista e desvios posturais corporais, principalmente cifose, escoliose e retificação de pescoço, sendo que o desenvolvimento dos desvios associa-se de maneira significativa com o aumento da faixa etária, da jornada de trabalho diário, do tempo de atuação profissional e conforme o gênero do indivíduo.

O exercício profissional obriga que cirurgiões-dentistas utilizem na execução de suas tarefas os membros superiores e estruturas adjacentes, sendo as mãos particularmente muito exigidas, frequentemente com repetitividade de um mesmo padrão de movimento, em virtude da atividade clínica, e compressão mecânica das estruturas localizadas na região, em função da inadequação dos instrumentos, levando à assunção de posturas incorretas por necessidade das técnicas operatórias e à utilização de força excessiva em virtude das características particulares de algumas patologias; além disso, na maioria dos casos, os profissionais atuam sob a pressão da máxima eficiência temporal (REGIS FILHO; LOPES, 1997).

As LERs/DORTs são consequências tardias do mau uso crônico de um conjunto mecânico delicado constituído pelos membros superiores e regiões adjacentes, seja pelo uso da força excessiva, por compressão mecânica, posturas desfavoráveis das articulações ou alta repetitividade; assinalamos que, como fator isolado, o esforço excessivo se mostra mais importante que os efeitos deletérios de cada uma das outras condições.

Observa-se pela literatura pesquisada evidências de que um número significativo de cirurgiões-dentistas são ou foram portadores de LERs/DORTs já perfeitamente diagnosticadas. Entretanto, esses relatos ou eram oriundos de amostras pequenas e não representativas ou provinham de estudos de casos com subgrupos, originando controvérsias e não permitindo afirmar, ainda, que o grupo profissional dos cirurgiões-dentistas reúne características que permitem incluí-lo no grupo de risco no desenvolvimento de LERs/DORTs.

No presente estudo procurou-se detectar se o cirurgiãodentista integra grupo profissional exposto a risco considerável no desenvolvimento de algum tipo de LER/DORT, já que certas condições inerentes às tarefas desses profissionais - aí consideradas força excessiva, posturas incorretas, alta repetitividade de um mesmo padrão de movimento e compressão mecânica dos tecidos, aliadas às características individuais estão presentes e acusam o perigo.

Por opção metodológica, somente aspectos biomecânicos foram abordados, excluindo-se do presente trabalho uma análise mais profunda de outros fatores relacionados com as LERs/DORTs de cirurgiões-dentistas, como os psicossociais, os decorrentes das interações com instrumentos, os biológicos e/ou genéticos individuais, a exposição a agentes externos químicos e/ou biofísicos, os legais e os de organização temporal do trabalho; tal opção determina os limites do presente estudo.

\section{MATERIAL E MÉTODOS}

O presente estudo utilizou biomecânica para buscar evidências de relação entre as tarefas executadas pelo cirurgião-dentista e as LERs/DORTs. Foram realizadas, com o auxílio do Laboratório de Biomecânica do Centro de Educação Física, Fisioterapia e Desportos da Universidade do Estado de Santa Catarina, análises biomecânicas 
compreendendo cinemetria e eletromiografia de quatro tarefas executadas cotidianamente por cirurgiões-dentistas generalistas, objetivando obter dados sobre os componentes neuromusculares determinantes desses movimentos humanos e os fenômenos mecânicos dos segmentos corporais envolvidos com as LERs/DORTs (ARAÚJO, 1998). Cinemetria e eletromiografia foram utilizadas, não simultaneamente, por serem ferramentas confiáveis e relativamente fáceis de manipular, sendo cada vez mais utilizadas e permitindo assim, a princípio, comparações entre diferentes experimentos.

As análises biomecânicas utilizando cinemetria e eletromiografia foram realizadas em quatro cirurgiões-dentistas, um de cada uma das especialidades relacionadas a seguir: a) periodontia - foram observados os movimentos relacionados com raspagem e curetagem sub e supragengival; b) endodontia - o procedimento observado foi endodontia de um dente unirradicular; c) cirurgia e traumatologia bucomaxilofaciais - nessa especialidade foram observados os procedimentos relacionados com uma exodontia ou extração dentária de um dente molar superior; e d) dentística restauradora - a tarefa observada foi a realização de uma restauração com o material restaurador amálgama em um dente molar inferior.

As coletas dos dados cinemáticos e a aquisição dos sinais eletromiográficos foram realizadas em situação real de trabalho, ou seja, em atendimento clínico de pacientes, e o tempo médio das intervenções de cada um dos quatro profissionais foi de uma hora cada.

Para Pece (1995), as atividades mais praticadas pelos cirurgiões-dentistas clínicos gerais são, em ordem decrescente: 1) dentística; 2) periodontia; 3) exodontia; e 4) endodontia. Dos cirurgiões-dentistas por ele pesquisados, $100 \%$ praticavam procedimentos relacionados com dentística e exodontia e $87 \%$ e $26 \%$, respectivamente, praticam procedimentos relacionados com periodontia e endodontia.

Outro aspecto importante a salientar é que no Brasil $86,2 \%$ dos cirurgiões-dentistas são generalistas, ou seja, realizam as quatro especialidades especificadas (PECE, 1995). Assim, a escolha destas especialidades se deveu, basicamente, ao fato de abrangerem os procedimentos odontológicos mais comumente realizados nas atividades de clínica geral, tanto privada como pública, englobando praticamente a totalidade dos movimentos exigidos no exercício da odontologia, como os de sensibilidade fina, caso da endodontia, ou os que exigem alguma força, a exemplo do que ocorre na exodontia.
Os profissionais que participaram das análises biomecânicas eram todos especialistas, devidamente registrados no Conselho Federal de Odontologia, com mais de dez anos no exercício da especialidade, destros e sem nenhuma manifestação, presente ou passada, dolorosa ou não, nos membros superiores, cintura escapular e pescoço em virtude da repetição de um mesmo padrão de movimento laboral. Todos eram do sexo masculino, tinham entre $30 \mathrm{e}$ 
Digitalizando-se uma série de pontos de coordenadas X, Y e Z conhecidas, o método Direct Linear Transformation (DLT) permite dimensionar qualquer outro objeto que esteja no volume calibrado; também se pode descrever um movimento com base nas sucessivas imagens do objeto e no intervalo de tempo entre elas. Neste caso, os movimentos realizados pelos profissionais foi reconstruído com base nas imagens das quatro câmeras, que estavam posicionadas de modo que, em relação ao centro da área de calibração, os eixos ópticos entre as câmeras 1 e 2 e entre as câmeras 3 e 4 formavam ângulos de $118,8^{\circ}$ e, entre as câmeras 2 e 3 e entre as câmeras 3 e 4 , ângulos de $68,2^{\circ}$.

O programa utiliza o método dos mínimos quadrados para determinar o melhor ajuste dos parâmetros de cada câmera, os quais se mantêm inalterados enquanto a posição da câmera, distância focal ou foco das lentes não são modificados. O programa oferece como opção a escolha do número de parâmetros que serão utilizados na reconstrução tridimensional dos objetos, sendo possível escolher 11, 12, 14 ou 16 parâmetros. Doze parâmetros são utilizados para corrigir a distorção simétrica das lentes, 13 e 14 são utilizados para corrigir distorções simétricas de lentes de quinta e sétima ordem e 15 e 16 são utilizados para corrigir distorções assimétricas das lentes.

O tratamento estatístico dos dados foi realizado a partir dos ângulos obtidos após a reconstrução em três dimensões (3D) obtendo-se médias angulares, erro e desvio padrão, variância da amostra, coeficiente de variação e valores angulares mínimos e máximos. Valores da amplitude angular dos segmentos corporais encontradas na literatura foram comparados com a média dos ângulos.

O local da coleta dos dados foi uma das clínicas do Curso de Graduação em Odontologia da Universidade Federal de Santa Catarina, adaptada para as exigências que o sistema requer, sendo que os pacientes foram previamente notificados dos procedimentos que foram realizados, com os quais concordaram assinarndo um termo de consentimento livre e esclarecido.

A coleta dos dados cinemáticos foi realizada adotandose os seguintes procedimentos :

1) Preparação do profissional: a) Comunicação prévia aos profissionais dos procedimentos de avaliação, os quais, após terem sido esclarecidos e concordarem, assinaram termo de consentimento;

b) Preparação dos profissionais para a realização da atividade com jalecos de cor não contrastante, evitando assim que refletissem a luz dos flash das câmeras, sendo que as mangas dos jalecos foram retiradas para otimizar a visualização dos marcadores afixados aos ombros dos profissionais; $\mathrm{e}$

c) Localização e marcação dos pontos anatômicos, de acordo com o modelo espacial adotado.

\section{2) Localização dos eixos articulares:}

A localização dos eixos articulares foi determinada através da projeção dos mesmos sobre a pele, conforme procedimentos descritos por Kalfhues (1971) e Riehle (1976), citados por Kraeski et al. (1999), conforme os valores apresentados na Tabela 1.

Com o auxílio desses valores procedeu-se à marcação dos eixos articulares nos profissionais. Para a marcação destes eixos foram utilizados marcadores revestidos com fita adesiva especial em formato de círculo com $1 \mathrm{~cm}$ de diâmetro.

\section{3) Aquisição das imagens:}

Para a aquisição das imagens, o profissional se posicionou no centro da área de calibração e a partir daí executou os procedimentos afetos à sua especialidade. O período de gravação compreendeu do inicio até o fim da atividade, sendo que a frequência de aquisição usada foi de $60 \mathrm{~Hz}$.

\section{4) Processamento dos dados:}

Com a finalidade de atender aos objetivos do presente estudo adotou-se o seguinte processamento dos dados:

a) Seleção das câmeras e determinação das características dos profissionais;

b) Determinação do modelo espacial utilizado;

c) Determinação do sistema de referência ou calibrador de eventos que representou o sistema de referência e erro relativo ao espaço-objeto e, igualmente, os eventos correspondentes à fase inicial e final das etapas: deslocamento do profissional até a mesa de instrumentos e seu retorno durante o procedimento em exame;

Tabela 1: Localização dos eixos articulares

\begin{tabular}{ll}
\hline \multicolumn{1}{c}{ Eixos articulares } & \multicolumn{1}{c}{ Posição } \\
\hline Articulação escapuloumeral & $4,9 \mathrm{~cm}$ do canto superior distal do acrômio \\
Articulação do cotovelo & $1,1 \mathrm{~cm}$ da fenda lateral proximal da articulação do cotovelo \\
Articulação do punho & $1,1 \mathrm{~cm}$ da ponta distal do processo estiloidal radial \\
Ponto na mão & Sobre a falange proximal do dedo médio \\
\hline
\end{tabular}


d) Aquisição da imagem do calibrador para posterior digitalização;

e) Imagem do calibrador digitalizado, sendo que o ponto preto no centro dos marcadores corresponde à digitalização;

f) Seleção das imagens de cada uma das câmeras para digitalização;

g) Imagem digitalizada, sendo que as imagens foram processadas no próprio sistema Peak Motus. A digitalização dos pontos referenciais foi automática e semiautomática, gerando arquivos de dados brutos, coordenadas brutas, coordenadas escalares e coordenadas transformadas, permitindo a determinação de: trajetória dos eixos articulares, velocidade linear, velocidade angular, aceleração linear e angular e ângulos;

h) Após a digitalização foram determinadas as variáveis a serem calculadas; $\mathrm{e}$

i) Imagem das quatro câmeras e modelo espacial em 3 dimensões (3D) representando virtualmente as proporções dos segmentos e as trajetórias dos eixos articulares realizadas pelo sujeito em estudo na amostra. hardware apresenta três componentes integrados: 1) componente processador de sinal; 2) componente de aquisição de dados; e 3) componente de vídeo.

O sub-sistema de software é composto de três secções: 1) interface do usuário; 2) sistema de operação; e 3) processo de digitalização dos sinais.

O Sistema AMLAB/1997 é um Instrumento Emulador Baseado em Computador que processa a entrada e a saída dos sinais em perfeita sincronização e independente do número de canais ou da frequência escolhida. Um sistema de quatro canais foi projetado para ser o eletromiógrafo, possibilitando o registro simultâneo da atividade elétrica de quatro músculos ou grupos musculares.

No presente estudo foram utilizados para cada canal ou grupo muscular dois eletrodos como eletrodos ativos de formato retangular, medindo cada um $10 \times 4 \mathrm{~mm}$, dispostos paralelamente com espaçamento de $10 \mathrm{~mm}$ entre eles. Os eletrodos foram colocados bem próximos dos pontos motores ou ventres de cada grupo muscular. O eixo mais longo dos eletrodos foi posicionado transversalmente às fibras musculares, sendo que os eletrodos proximais foram ligados às entradas inversoras dos amplificadores diferenciais, enquanto os distais foram conectados às entradas não inversoras.

\subsection{Eletromiografia}

Devido à pequena amplitude dos potenciais elétricos resultantes da atividade muscular, que são de no máximo algumas unidades de milivolts, estes só podem ser captados se uma aparelhagem adequada for utilizada. Essa aparelhagem deve

\section{s LERs/DORTs representam a conseqüência tardia do
mau uso crônico de um delicado conjunto mecânico}

\section{que são os membros superiores e regiões adjacentes}

apresentar características como: a) elevada impedância de entrada - no mínimo alguns megaohms; b) alta amplificação de tensão - pelo menos 1.000 vezes; e c) isolamento elétrico (ARAÚJO, 1998).

Para aquisição dos sinais eletromiográficos foi utilizado um sistema denominado Associative Measurement Laboratory - AMLAB/1997 especialmente desenvolvido para pesquisas com seres humanos.

O sistema permite trabalhar com biopotências como as oriundas das fibras musculares e opera com uma frequência de aquisição de $1.000 \mathrm{~Hz}$, ou seja, um ponto a cada milissegundo, com ganho de tensão de 4.000 vezes e uma impedância de entrada maior que $1.012 \Omega$. O sistema trabalha segundo programação orientada por objeto, onde o instrumento é projetado na tela do computador de acordo com os recursos disponíveis nas bibliotecas utilizadas para as diferentes necessidades.

O AMLAB/1997 é composto de um sub-sistema de hardware e um sub-sistema de software. O sub-sistema de
Um eletrodo de referência ou terra foi colocado na extremidade superior do externo e entre manúbrio e incisura jugular. A pele do profissional sob os eletrodos foi preparada para a coleta dos sinais eletromiográficos valendo-se de procedimento padrão, ou seja, raspagem dos pêlos e limpeza da região com álcool de uso doméstico (BASMANJIAN; DeLUCA, 1985, citados por LOSS et al. 1999). Para normalização do sinal eletromiográfico foram realizadas uma série de contrações voluntárias máximas (CVM) em isometria, durante cinco segundos, envolvendo os grupos musculares selecionados.

Durante as atividades os sinais eletromiográficos foram captados em ciclos com duração de 15 segundos cada um. Os dados adquiridos, após serem processados em seu sistema de origem, foram então transportados em formato ASCII para um software de tratamento de dados, onde foram selecionadas todas as curvas de interesse para a interpretação integrada.

O software utilizado para aquisição de dados processados na origem foi o Sistema de Aquisição de Dados 
(SAD), completamente desenvolvimento no Laboratório de Medições Mecânicas da Universidade Federal do Rio Grande do Sul, que além de possuir diversas ferramentas matemáticas para tratamento de sinais permite a aquisição de dados para uma grande variedade de placas conversoras analógicas/digitais existentes no mercado (LOSS et al. 1999).

O software permite trabalhar com diversos arquivos abertos simultaneamente, possibilitando a livre importação de curvas entre arquivos e facilitando a troca de informações. Cada arquivo do programa têm diversas curvas com sinais adquiridos e filtrados, ou seja, processados de todas as formas que o programa permite, sendo que as operações são feitas a partir de comandos existentes em menus tipo pull-down ou, ainda, através de teclas de atalho (LOSS et al. 1999).

Os músculos analisados foram o trapézio, o deltóide e os grupos musculares dos flexores e extensores do carpo. A escolha dos músculos analisados pode ser atribuída, primeiramente, a sua posição anatômica superficial, que permite o uso da eletromiografia de superfície, e, também, por estarem associados com os movimentos executados na prática odontológica (LOSS et al. 1999).

\section{RESULTADOS E DISCUSSÃO}

\subsection{Cinemetria}

Após a aquisição dos dados cinemáticos foram selecionados intervalos nos quais foi realizada a análise das imagens. Os tempos de aquisição das imagens se situaram entre 16,67 e $25,00 \%$ do tempo total do evento, sendo os intervalos, portanto, bastante significativos para as análises dos dados coletados.

Os dados cinemáticos foram obtidos nos segmentos corporais punho direito, cotovelo direito, cabeça em relação ao plano $\mathrm{X}$ e ombro em relação aos planos XY, XZ e YX, e estão representados em forma de gráfico, sendo que os movimentos que ocorreram fora da área de calibração se encontram entre barras verticais. Foram realizadas um mínimo de 2.980 e um máximo 8.936 medições angulares de cada segmento corporal analisado, sendo que a média foi de 4.686 medições. A literatura pesquisada permitiu classificar os ângulos posturais em níveis de risco que são apresentados na Tabela 2 e onde se encontram os ângulos médios observados no presente estudo relativo a diversos segmentos corporais de interesse para as LERs/DORTs.

A classificação em níveis de risco foi importante, pois limites aceitáveis de amplitudes angulares para as diferentes posturas podem ser traçados. Entretanto é necessário salientar que não é pelo fato de se encontrar alguma flexão ou abdução do braço acima do nível dos ombros, ou mesmo flexão, extensão ou desvio ulnar do punho no ciclo da tarefa que se caracteriza o risco. O risco é caracterizado pela frequência desse tipo de ação, ou seja, pela repetitividade, pela sua manutenção, caracterizando esforço estático ou, ainda, pelo uso de força excessiva (COUTO; NICOLETTI; LECH, 1998).

Observa-se que praticamente em todos os segmentos se encontram ângulos médios em amplitudes angulares consideradas de risco médio, sendo que para cabeça se encontra valores considerados de risco alto. Os dados cinemáticos obtidos estão de acordo com estudo realizado por

Tabela 2: Comparação da classificação das posturas em níveis de risco
1) Bramson, Smith e Romagnoli (1998); 2) Grandjean (1998); 3) Bernard (1997);
Regis Filho, Michels e Sell*

4) Couto, Nicoletti e Lech (1998); 5) Grandjean (1998)

Procedimento

Postura

Exodontia Raspagem e Restauração Endodontia curetagem com amálgama

1) Flexão do punho: 0 a $15^{\circ}$ risco baixo, $>15$ a $45^{\circ}$ risco médio e $>45^{\circ}$ risco alto.

1) Extensão do punho: 0 a $15^{\circ}$ risco baixo, $>15$ a $45^{\circ}$ risco médio e $>45^{\circ}$ risco alto.

1) Desvio ulnar do punho: 0 a $20^{\circ}$ risco baixo, $>20$ a $30^{\circ}$ risco médio e $>31^{\circ}$ risco alto.

1) Desvio radial do punho: 0 a $10^{\circ}$ risco baixo, $>10$ a $20^{\circ}$ risco médio e $>20^{\circ}$ risco alto.

2) Flexão do cotovelo: 0 a $90^{\circ}$ risco baixo, $>90$ a $120^{\circ}$ risco médio e $>120^{\circ}$ risco alto.

3) Flexão do ombro: 0 a $30^{\circ}$ risco baixo, $>30$ a $60^{\circ}$ risco médio e $>60^{\circ}$ risco alto.

3) Abdução do ombro: 0 a $30^{\circ}$ risco baixo, $>30$ a $60^{\circ}$ risco médio e $>60^{\circ}$ risco alto.

4) Rotação medial do ombro: 0 a $10^{\circ}$ risco baixo, $>10$ a $45^{\circ}$ risco médio e $>45^{\circ}$ risco alto.

5) Flexão da cabeça: 0 a $10^{\circ}$ risco baixo, $>10$ a $30^{\circ}$ risco médio e $>30^{\circ}$ risco alto.

\begin{tabular}{cccc}
$20.9(159,1)$ & $22.4(157,6)$ & $13.5(166,5)$ & $24.9(155,1)$ \\
- & - & - & - \\
32 & 22 & 45 & 31 \\
- & - & - & - \\
81.2 & 82.7 & 54.8 & 74.9 \\
29.7 & 14.9 & 35.0 & 18.1 \\
30.4 & 22.3 & 25.0 & 37.7 \\
42.7 & 61.4 & 42.2 & 45.3 \\
45,8 & 46,1 & 30,3 & 47,5 \\
\hline
\end{tabular}

*Resultados obtidos no presente estudo. 
Sayegh et al. (2005) com profissionais libaneses - o qual indicou presença de dor na coluna lombar em $66 \%$ dos casos, na coluna cervical em $59 \%$, e no ombro e braço em $62 \%$ dos entrevistados - e com o trabalho de Leggat e Smith (2006), que indicou que $89,1 \%$ dos cirurgiões-dentistas pesquisados tinham experimentado algum sintoma de LER/DORT nos últimos 12 meses, sendo que $57,5 \%$ indicaram o pescoço como a região dolorosa, 53,7\%, a região baixa do dorso, e $53,3 \%$, o ombro.

As amplitudes angulares encontradas no trabalho do endodontista e classificadas de risco médio e alto em praticamente todos os segmentos corporais (excessão feita ao ombro em flexão, que foi classificado de risco baixo), estão de acordo com o trabalho desenvolvido por Michelotto, Silva Junior e Sydney (2005) com endodontista, o qual utilizou Análise Ergonômica do Trabalho e o Método OWAS, encontrando posturas constrangedoras para o organismo durante o atendimento clínico.

Na tabela 3 estão os valores obtidos por Bramson, Smith e Romagnoli (1998) para alguns procedimentos da prática odontológica comparados com os do presente estudo. Embora as tarefas executadas não sejam semelhantes, esses autores observaram procedimentos odontológicos realizados em amplitudes angulares consideradas de risco médio.

As posturas observadas na análise cinemática e classificadas de amplitude angular de risco alto para as regiões da

Tabela 3: Comparação da amplitudes dos movimentos angulares

\begin{tabular}{|c|c|c|c|}
\hline & \multirow[t]{2}{*}{ Postura } & \multicolumn{2}{|l|}{ Angular } \\
\hline & & Procedimentos & Angulação \\
\hline \multirow{8}{*}{ 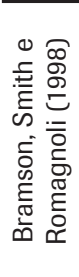 } & Flexão do punho & Sondagem & $6,25^{\circ}$ \\
\hline & & Raspagem & $13,67^{\circ}$ \\
\hline & & Polimento & $12,76^{\circ}$ \\
\hline & & Fio dental & $14,33^{\circ}$ \\
\hline & Desvio ulnar & Sondagem & $13,63^{\circ}$ \\
\hline & & Raspagem & $18,15^{\circ}$ \\
\hline & & Polimento & $17,31^{\circ}$ \\
\hline & & Fio dental & $20,79^{\circ}$ \\
\hline \multirow{10}{*}{ 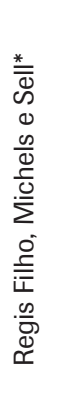 } & Flexão do punho & Exodontia & $20,9^{\circ}$ \\
\hline & & Raspagem e curetagem & $22,4^{\circ}$ \\
\hline & & Condensação & $13,5^{\circ}$ \\
\hline & & amálgama & $24,9^{\circ}$ \\
\hline & & $\begin{array}{l}\text { Instrumentação do } \\
\text { canal radicular }\end{array}$ & \\
\hline & Desvio ulnar & Exodontia & $32^{\circ}$ \\
\hline & & Raspagem e curetagem & $22^{\circ}$ \\
\hline & & Condensação & $4^{\circ}$ \\
\hline & & amálgama & $31^{\circ}$ \\
\hline & & $\begin{array}{l}\text { Instrumentação do } \\
\text { canal radicular }\end{array}$ & \\
\hline
\end{tabular}

${ }^{*}$ Resultados obtidos no presente estudo. cabeça e punho e de risco médio para alto na região do ombro são compatíveis com os valores encontrados para a incidência de LERs/DORTs nas regiões de pescoço, punho e ombro.

\subsection{Eletromiografia}

Os valores dos sinais eletromiográficos obtidos foram transformados em percentuais em relação à contração voluntária máxima (CVM) e classificados de risco baixo, médio oue alto para possibilitar a comparação com a literatura.

Na tabela 4 estão os percentuais da contração voluntária máxima $(\mathrm{CVM})$ obtidos através da eletromiografia de quatro grupos musculares, a qual foi realizada nos quatro profissionais (cirurgião bucomaxilofacial, periodontista, especialista em dentística e endodontista) durante a execução de diversos procedimentos odontológicos; os dados obtidos foram comparados aos níveis de exigência muscular da contração voluntária máxima em vários procedimentos odontológicos. A análise dos percentuais encontrados indicou que em praticamente todas as tarefas investigadas o profissional corre risco alto de desenvolver algum tipo de LER/DORT.

Os percentuais de exigência muscular encontrados para os músculos deltóide e trapézio estão em perfeito acordo com o que foi observado por Pece (1995) para o procedimento exodontia. No presente estudo, apenas a atividade condensação do amálgama apresentou risco baixo para o músculo deltóide. Os elevados níveis de exigência muscular encontrados no estudo eletromiográfico juntamente com a postura inadequada observada durante a cinemetria, e os valores resultantes da análise epidemiológica, em que os profissionais pesquisados apresentaram incidência significativa de algum sinal ou sintoma das LERs/DORTs, são mais um indicativo de que os cirurgiões-dentistas estão expostos a desenvolver algumas dessas patologias.

\section{CONCLUSÕES}

No presente estudo, a análise dos dados cinemáticos revela que as principais atividades realizadas pelos cirurgiões-dentistas submetem duas ou mais regiões do corpo a posturas consideradas de risco médio e alto de ocorrência de LERs/DORTs, bem como a análise dos sinais eletromiográficos indicou um grande comprometimento dos grupamentos musculares flexores e extensores do carpo e do trapézio, o que explica a maior incidência das LERs/DORTs nas regiões de ombro/braço e punho/mão.

Por outro lado, a análise biomecânica permite com provar o que pesquisas utilizando estudos epidemiológicos transversais e Análise Ergonômica do Trabalho já indicavam: que os cirurgiões-dentistas formam um grupo 
Tabela 4: Comparação dos níveis de exigência muscular em relação a CVM durante vários procedimentos

\begin{tabular}{|c|c|c|c|c|c|}
\hline & \multirow[t]{2}{*}{ Prrocedimento } & \multicolumn{4}{|c|}{ Grupo Muscular } \\
\hline & & Flexores do carpo & Extensores do carpo & Deltóide & Trapézio \\
\hline \multirow{4}{*}{$\begin{array}{l}\text { Branson, Smith e } \\
\text { Romagnoli (1998) }\end{array}$} & Sondagem & 17,6 & 12,8 & & \\
\hline & Raspagem & 17,6 & 15,7 & & \\
\hline & Polimento & 18,8 & 22,6 & & \\
\hline & Fio dental & 20,2 & 15,3 & & \\
\hline \multirow{4}{*}{$\begin{array}{l}\text { Regis Filho, Michels } \\
\text { e Sell* }\end{array}$} & Exodontia & 68,94 & 72,53 & 32,24 & 25,26 \\
\hline & $\begin{array}{l}\text { Raspagem e } \\
\text { curetagem }\end{array}$ & 38,88 & 17,38 & 13,17 & 32,41 \\
\hline & $\begin{array}{l}\text { Condensação } \\
\text { amálgama }\end{array}$ & 15,94 & 39,80 & 5,62 & 41,68 \\
\hline & $\begin{array}{l}\text { Instrumentação do } \\
\text { canal radicular }\end{array}$ & 23,29 & 79,27 & 88,90 & 77,49 \\
\hline Pece (1995) & & & & Grande & Muito grande \\
\hline
\end{tabular}

Risco Baixo $=0$ a 10\% da CVM, risco médio $=>10$ a 20\% da CVM e risco alto $=>20 \%$ da CVM (BERNARD, 1997)

*Resultados obtidos no presente estudo.

profissional exposto a risco considerável de adquirir algum tipo de LER/DORT, desde que certos fatores inerentes às tarefas profissionais aí consideradas, como força excessiva, postura incorreta, alta repetitividade de um mesmo padrão de movimento e compressão mecânica dos tecidos, aliadas às características individuais, estejam presentes.

Os resultados obtidos indicam, ainda, que a maioria dos cirurgiões-dentistas, em virtude da utilização de instrumentos que não obedecem a critérios ergonômicos e da realização de tarefas inadequadamente prescritas, entre outros fatores, enfrenta condições adversas de trabalho, onde dor e desconforto estão presentes.

A metodologia empregada no presente estudo (baseada na análise biomecânica com cinemetria e eletromiografia) mostrou-se confiável, exequível e adequada, pois permitiu a aquisição de um volume significativo de informações, possibilitando conhecer o cirurgião-dentista e seu trabalho. Entretanto deve-se ressaltar que o método é dispendioso ou relativamente caro, não sendo indicado para estudos mais extensos.

Mais pesquisas que possibilitem o redesenho das tarefas são necessárias, permitindo assim a redução de tensões biomecânicas sobre os membros superiores e as regiões adjacentes e incentivando a criação de instrumentos que atendam a requisitos ergonômicos. $\mathrm{O}$ instrumento não deve ser simplesmente efetivo para a realização da tarefa para a qual foi concebido, ele também precisa ser considerado adequado pelo usuário em termos de balanço, peso, forma, vantagem biomecânica, sensibilidade ao contato e modo de utilização, minimizando o desenvolvimento do processo de fadiga e da tensão que se acumula ao longo do dia de trabalho.

Finalizando, a comprovação da associação das LERs/ DORTs com as tarefas executadas por esse grupo profissional, a qual até então não era praticamente citado na literatura sugere que pesquisas mais intensas devam ser realizadas com a finalidade de aprofundar o debate, principalmente aquelas que abordam fatores psicossociais, culturais e de organização temporal das tarefas, e também aquelas afetas à antropometria, a fim de possibilitar a confecção de instrumentos e equipamentos mais adequados às dimensões corporais dos profissionais brasileiros e por fim utilizando a dinamometria para mensurar a força e a pressão exercidas sobre os instrumentos de uso frequente na prática odontológica.

\section{Artigo recebido em 5/6/2006}

Aprovado para publicação em 28/6/2009 


\section{REFERÊNCIAS}

ARAÚJO, R. C. Utilização da eletromiografia na análise biomecânica do movimento humano. São Paulo, 1998. 153 p. Tese (Doutorado em Educação Física) - Universidade de São Paulo - USP.

ASSUNÇÃO, A. A. Sistema músculo-esquelético: lesões por esforços repetitivos (LER.) In: MENDES, R. Patologia do trabalho. Rio de Janeiro: Atheneu, 1997. p. 173-212.

BARNHART, S. et al. Carpal syndrome among ski manufacturing workers. Scandinavian Journal of Work, Environment and Health, v. 17, n. 1, p. 46-53, 1991.

BARREIRA, T. H. C. Abordagem ergonômica na prevenção da LER. Revista Brasileira de Saúde Ocupacional, v. 22, n. 84, p. 51-60, 1994.

BAUER, M. E. Carpal tunnel syndrome: an occupational risk to the hygienist. Journal of Dental Hygiene, v. 59, n. 5, p. 218-221, 1985.

BERNARD, B. P. Musculoskeletal disorders (MSDs) and workplace factors. 1997. Disponível em: <http://www.cdc.gov/niosh>

BRAMSON, J. B.; SMITH, S.; ROMAGNOLI, G. Evaluating dental office ergonomic risk factors and hazards. Journal of the American Dental Association, v. 129, n. 2, p.174-183, 1998.

CÂNDIDO, L.; BITTENCOURT, M. Z.; REGIS FILHO, G. I. Lesões por esforços repetitivos em cirurgiões-dentistas: um estudo de caso - Cisto sinovial de punho. Jornal Brasileiro de Clínica Odontológica Integrada, v. 7, n. 42, p. 463-466, 2003.

CODO, W.; ALMEIDA, M. C. C. G. LER: diagnóstico, tratamento e prevenção. Uma abordagem interdisciplinar. Petrópolis: Vozes, 1997. 355 p.

COUTO, H. A. Doenças profissionais: guia prático de tenossinovites e outras doenças de origem ocupacional. Belo Horizonte: Asta Médica, 1994. 9 p.

COUTO, H. A.; NICOLETTI, S. J.; LECH, O. Como gerenciar a questão das $\angle E R / D O R T$ : lesões por esforços repetitivos/distúrbios osteomusculares relacionados ao trabalho. Belo Horizonte: Ergo, 1998. 438p

CUNHA, C. E. G. et al. Ler: Lesões por Esforços Repetitivos: revisão. Revista Brasileira de Saúde Ocupacional, v. 20, n. 76, p. 47-59, 1992.
ECCLES, J. D.; DAVES, M. H. A study of operating positions in conservative dentistry. Dental Practitioner and Dental Record, v. 21, n. 3, p. 221-225, 1971.

ECCLES, J. D.; POWELL, M. The health of dentists. British Dental Journal, v. 123, n. 12, p. 379-387, 1967.

ERDIL, M.; DICKERSON, O. B.; GLACKIN, E. Cumulative trauma disorders of the upper extremity. In: ZENZ, C. Occupational Medicine. 3 ed. St. Louis: Mosby Year Book, 1994. p. 48-64.

FACCI, R. C. et al. A síndrome do excesso de uso. Proteção, v. 1, n. 6, p. 41-44, 1989.

FADEL, M. A. V. As lesões por esforço repetitivo em cirurgiões-dentistas: síndrome do impacto. Uma abordagem ergonômica. In: ENCONTRO NACIONAL DE ENGENHARIA DA PRODUÇÃO - ENEGEP, 26, 2006a. Anais...

FADEL, M. A. V. Lesões por esfoço repetitivo em cirurgiões-dentistas: um estudo de caso. Dedo em gatilho. Revista da Associação Paulista de Cirurgiões-Dentistas, v. 60, n. S, p. 189, 2006b.

FERGUSON, D. A. RSI: putting the epidemic to rest. Medical Journal of Australia, v. 147, p. 213-214, 1987.

FEUERSTEIN, M.; FITZGERALD, T. Biomechanical factors affecting upper extremity cumulative trauma disorders in sign language interpreters. Journal of Occupational and Environmental Medicine, v. 34, n. 3, p. 257-264, 1992.

GRANDJEAN, E. Manual de ergonomia: adaptando o trabalho ao homem. Porto Alegre: Bookman, 1998. 338 p.

HOLTZHAUSEN, T. Carpal tunnel syndrome: a new occupational hazard for the oral hygienist. Occupational hazards, v. 76, n. 6, p. 33-34, 1986.

Instituto Nacional do Seguro Social - INSS. Diário Oficial da União, n. 158, p. 26-34, 1998.

JETZER, T. C. Use of vibration testing in the early evaluation of workers with carpal tunnel syndrome. Journal of Occupational and Environmental Medicine, v. 33, n. 2, p. 117-120, 1991.
KRAESKI, M. H. et al. Reconstrução 3D: estudo de erros na obtenção das coordenadas espaciais. In: CONGRESSO BRASILEIRO DE BIOMECÂNICA, 8, 1999. Anais... Florianópolis: Sociedade Brasileira de Biomecânica, 1999. p. 351-356.

LAWRENCE, J. S. Rheumatic diseases. International Labour office Encyclopaedia of Occupational Healty and Safety, v. 2, n. 1, p. 1233-1234, 1972.

LEGGAT, P. A.; SMITH, D. R. Musculoskeletal disorders self-reported by dentists in Queensland, Australia. Australian Dental Journal, v. 51, n. 4, p. 324-327, 2006.

LOPES, A.; VILLANACCI NETO, R. A síndrome do túnel carpal: um risco profissional para o cirurgião-dentista. Revista da Associação Paulista de Cirurgiões-Dentistas, v. 48, n. 6, p. 1545-1552, 1994.

LOSS, J. F. et al. Análise de movimentos nas articulações do ombro, cotovelo e rádio-ulnar: um estudo eletromiográfico. In: CONGRESSO BRASILEIRO DE BIOMECÂNICA, 8, 1999. Anais... Florianópolis: Sociedade Brasileira de Biomecânica, 1999. p. 493-498.

MACIEL, A. C. C.; FERNANDES, M. B.; MEDEIROS, L. S. Prevalência e fatores associados à sintomatologia dolorosa entre profissionais da indústria têxtil. Revista Brasileira de Epidemiologia, v. 9, n. 1, p. 94-102, 2006.

MACIEL, R. H. Considerações gerais sobre o trabalho de digitação. Revista Brasileira de Saúde Ocupacional, v. 13, n. 50, p. 37-40, 1985.

MARTINS, C. A.; FADEL, M. A. V.; REGIS FILHO, G. I. Lesões por esforços repetitivos em cirurgiões-dentistas: um estudo de caso. Síndrome do túnel carpal. Jornal Brasileiro de Clínica Odontológica Integrada, v. 7, n. 41, p. 363-367, 2003.

McKEAG, A. Overuse injuries. Journal of Primary Care, v. 18, n. 4, p. 851-865, 1991.

MICHELOTTO, A. L.; SILVA JUNIOR, J. A.; SYDNEY, G. B. Uma abordagem ergonômica postural durante o tratamento endodôntico. Jornal Brasileiro de Clínica Odontológica Integrada, v. 9, n. 50-51, p. 192-198, 2005. 
MUROFUSE, N. T.; MARZIALE, M. H.P.Doenças do sistema osteomuscular em trabalhadores de enfermagem. Revista Latino-Americana de Enfermagem, v. 13, n. 3, p. 364-373, 2005.

NOGUEIRA, D. P. Riscos ocupacionais de dentistas e sua prevenção. Revista Brasileira de Saúde Ocupacional, v. 11, n. 41, p. 14-16, 1983.

OLIVEIRA, C. R. Lesão por esforço repetitivo (LER). Revista Brasileira de Saúde Ocupacional, v. 19, n. 73, p. 59-85, 1991

PECE, C. A. Z. Concepção ergonômica, desenvolvimento e otimização de um fórceps odontológico: proposta de nova sistemática exodôntica. São José dos Campos, 1995. 168 p. Dissertação (Mestrado em Mecânica dos Sólidos e Estruturas) - Instituto Tecnológico de Aeronáutica - ITA.

PICOLOTO, D.; SILVEIRA, E. Prevalência de sintomas osteomusculares e fatores associados em trabalhadores de uma indústria metalúrgica de Canoas - RS. Revista Ciência e Saúde Coletiva, v. 13, n. 2, p. 507-516, 2008.
PIETROBON, L.; REGIS FILHO, G. I. Cifoescoliose em cirurgiões-dentistas: uma abordagem ergonômica. In: REUNIÃO ANUAL DA SOCIEDADE BRASILEIRA DE PESQUISA ODONTOLÓGICA, 23, 2006. Anais... Brazilian Oral Research, v. 20, p. 286-286, 2006.

REGIS FILHO, G. I.; LOPES, M. C. Aspectos epidemiológicos e ergonômicos de lesões por esforço repetitivo em cirurgiões-dentistas. Revista da Associação Paulista de CirurgiõesDentistas, v. 51, n. 5, p. 469-475, 1997.

REGIS FILHO, G. I.; MICHELS, G.; SELL, I. Lesões por esforços repetitivos/distúrbios osteomusculares relacionados ao trabalho em cirurgiões-dentistas. Revista Brasileira de Epidemiologia, v. 9, n. 3, p. 346-359, 2006.

ROCHA, L. E. M.; PAES, E. M.; SOBANIA, L. C. Lesões por esforço de repetição: análise em 166 digitadores de um centro de computação de dados. Revista Brasileira de Ortopedia, v. 21, n. 4, p. 115-119, 1986.
SANTOS FILHO, S. B. Prevalência de dor osteomuscular de membros superiores em cirurgiões-dentistas do serviço público de Belo Horizonte: contribuição no debate sobre os distúrbios osteomusculares relacionados ao trabalho (DORT). Minas Gerais, 1998. 30 p. Dissertação (Mestrado em Saúde Pública) - Universidade Federal de Minas Gerais - UFMG.

SAQUY, P. C.; PÉCORA, J. D. Orientação profissional em odontologia. São Paulo: Editora Santos, 1996. 67 p.

SAYEGH, G. M. et al. Musculo-skeletal problems among Lebanese dental surgeons: occurrence and risk factors. Journal Medical Libanais, v. 53, n. 1, p. 21-7, 2005.

SILVERSTEIN, B. A.; FINE, L. J.; ARMSTRONG, T. J. Occupational factors and the carpal tunnel syndrome. American Journal of Industrial Medicine, v. 11, n. 3, p. 343-358, 1987.

\section{SOBRE OS AUTORES}

\section{Gilsée Ivan Regis Filho}

Universidade Federal de Santa Catarina - UFSC

Florianópolis, SC, Brasil

E-mail: gregis@brturbo.com.br

\section{Glaycon Michels}

Universidade Federal de Santa Catarina - UFSC Florianópolis, SC, Brasil

E-mail: drglaycon@hotmail.com

\section{Ingeborg Sell}

Universidade Federal de Santa Catarina - UFSC

Florianópolis, SC, Brasil

E-mail: ingeborg.sell@gmail.com 